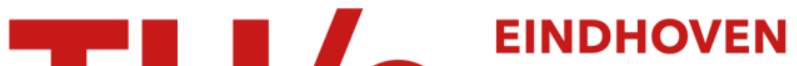 UNIVERSITY OF TECHNOLOGY
}

\section{Binaural detection with spectrally nonoverlapping signals and maskers: evidence for masking by aural distortion products}

\section{Citation for published version (APA):}

Heijden, van der, M. L., Trahiotis, C. T., Kohlrausch, A. G., \& Par, van de, S. L. J. D. E. (1997). Binaural detection with spectrally nonoverlapping signals and maskers: evidence for masking by aural distortion products. Journal of the Acoustical Society of America, 102(5. pT. 1), 2966-2972. https://doi.org/10.1121/1.420351

DOI:

10.1121/1.420351

Document status and date:

Published: 01/01/1997

\section{Document Version:}

Publisher's PDF, also known as Version of Record (includes final page, issue and volume numbers)

\section{Please check the document version of this publication:}

- A submitted manuscript is the version of the article upon submission and before peer-review. There can be important differences between the submitted version and the official published version of record. People interested in the research are advised to contact the author for the final version of the publication, or visit the $\mathrm{DOI}$ to the publisher's website.

- The final author version and the galley proof are versions of the publication after peer review.

- The final published version features the final layout of the paper including the volume, issue and page numbers.

Link to publication

\section{General rights}

Copyright and moral rights for the publications made accessible in the public portal are retained by the authors and/or other copyright owners and it is a condition of accessing publications that users recognise and abide by the legal requirements associated with these rights.

- Users may download and print one copy of any publication from the public portal for the purpose of private study or research.

- You may not further distribute the material or use it for any profit-making activity or commercial gain

- You may freely distribute the URL identifying the publication in the public portal.

If the publication is distributed under the terms of Article 25fa of the Dutch Copyright Act, indicated by the "Taverne" license above, please follow below link for the End User Agreement:

www.tue.nl/taverne

Take down policy

If you believe that this document breaches copyright please contact us at:

openaccess@tue.nl

providing details and we will investigate your claim. 


\title{
Binaural detection with spectrally nonoverlapping signals and maskers: Evidence for masking by aural distortion products
}

\author{
Marcel van der Heijden and Constantine Trahiotis \\ Surgical Research Center, Department of Surgery (Otolaryngology) and Center for Neurological Sciences, \\ University of Connecticut Health Center, Farmington, Connecticut 06030 \\ Armin Kohlrausch and Steven van de Par \\ Institute for Perception Research (IPO), P.O. Box 513, NL-5600 MB Eindhoven, The Netherlands
}

(Received 9 January 1997; accepted for publication 24 July 1997)

\begin{abstract}
Thresholds were measured for diotic tonal signals in the presence of interaurally delayed bands of Gaussian noise. When the signal frequency was $525 \mathrm{~Hz}$, the spectrum of the noise was either below (highest frequency, $450 \mathrm{~Hz}$ ) or above (lowest frequency, $600 \mathrm{~Hz}$ ) the frequency of the signal. When the signal frequency was $450 \mathrm{~Hz}$, the spectrum of the noise was always above the signal frequency (lowest frequency, $600 \mathrm{~Hz}$ ). Signals had a 250-ms duration and were temporally centered within the $300-\mathrm{ms}$ long bursts of noise. The spectrum level of the noise was $60 \mathrm{~dB}$. Thresholds obtained in all three conditions varied essentially sinusoidally with the interaural delay of the noise. For signals below the spectrum of the noise, the periodicities within the data were close to, but not identical with, the periodicities of the signals. This outcome is discussed in terms of masking produced by aural distortion products stemming from interactions within the bands of noise [cf. van der Heijden and Kohlrausch, J. Acoust. Soc. Am. 98, 3125-3134 (1995)]. For signals above the spectrum of the noise, the periodicities in the data suggested that masking was produced by components within the band of noise. Patterns within the data are also discussed in terms of limitations concerning the magnitude of external delays that can be matched by internal delays that are incorporated in modern models of binaural processing. (C) 1997 Acoustical Society of America. [S0001-4966(97)03211-6]

PACS numbers: 43.66.Pn, 43.66.Dc [JWH]
\end{abstract}

\section{INTRODUCTION}

In a recent study, van der Heijden and Kohlrausch (1995) showed that aural distortion products evoked by interactions among the components of a band of noise determined the detectability of a tonal signal whose frequency was below the spectral region of the noise. The level of the noise was low enough to preclude similar effects due to remote masking (e.g., Bilger and Hirsh, 1956). Van der Heijden and Kohlrausch found that the distortion products responsible for their "downward" masking effects had level-dependent phases that were similar to those found with odd-order distortion products such as the cubic difference tone (e.g., Goldstein, 1967). In addition, van der Heijden and Kohlrausch found that aural distortion products appeared to play no role when the frequency of the signal was above the spectral region of the noise (an "upward" masking condition). Their findings are consistent with well-known physiological and psychophysical observations concerning distortion products.

It occurred to us that a binaural paradigm could provide a further test of the ability of auditory distortion products to produce "downward" masking. The challenge was to differentiate between masking effects produced by components of a noise stimulus per se and masking effects produced by internally generated distortion products. Our strategy was to employ interaural delays of a band of noise and to use periodicities within the data to determine whether detectability was due to: (1) components within the band of noise, in this case remote from the frequency of the signal; or (2) distor- tion products having spectral components in the same frequency region as the signal. Said differently, we attempted to use interaural delays of a band of noise to separate offfrequency masking (i.e., masking due to spectral components spectrally distant to the signal frequency) from on-frequency masking (i.e., masking due to distortion products adjacent to and/or overlapping with the signal frequency).

Our expectation was that, if masking were "onfrequency" due to distortion products, then the pattern of thresholds, as a function of interaural delay of the noise, would reflect the frequency of the signal. That type of outcome was reported in the classic studies of Rabiner et al. (1966) and Langford and Jeffress (1964), who measured the detectability of tonal signals masked by Gaussian noise having components in the same spectral region as the signal. For the type of on-frequency masking which they studied, the data showed oscillations at the frequency of the signal as the masking noise was interaurally delayed.

To our knowledge, there are no data available concerning how binaural detectability varies with interaural delay when signal and masker occupy different spectral regions. We are aware of only two studies dealing with binaural processing in "off-frequency" conditions. McFadden et al. (1972) measured masking-level differences in a tone-on-tone paradigm with the signal placed spectrally above or below the sinusoidal masker and Hall et al. (1983) measured binaural detection in a notched-noise experiment. Neither of those two studies incorporated stimulus conditions involving interaural delays. Therefore, the data obtained in those studies may not directly bear on issues of interest here. Conse- 
quently, what the patterning of thresholds is as a function of delays in the maskers in the off-frequency case is an open question, which the data obtained in this investigation will help answer.

\section{METHOD}

Detection thresholds were determined using a threeinterval forced-choice adaptive procedure (Levitt, 1971). Each trial consisted of three 300-ms observation intervals each separated by $200 \mathrm{~ms}$. The signal occurred randomly, with equal a priori probability in each of the three intervals. The listeners were provided with correct-answer feedback via a computer terminal. Each estimation of threshold began with the signal being easily detectable. The level of the signal was decreased after two consecutive correct responses and was increased after each incorrect response. This procedure tracked the $70.7 \%$ correct point of the psychometric function. The step size was $8 \mathrm{~dB}$ at the beginning of each run, was reduced to $4 \mathrm{~dB}$ after the second reversal, and to 2 $\mathrm{dB}$ after the fourth reversal. Ten more reversals were obtained using 2-dB steps. An estimate of threshold was obtained by calculating the median of the levels over the ten last reversals. Final thresholds are the average of three such estimates.

Thresholds were obtained for tonal signals in the presence of a band of noise. In different conditions, the frequency of the signal was either below or above the frequencies composing the band of noise. When the band of noise had components ranging from 600 to $1100 \mathrm{~Hz}$, the frequency of the signal was either 450 or $525 \mathrm{~Hz}$. When the band of noise had components ranging from 0 to $450 \mathrm{~Hz}$, the frequency of the signal was $525 \mathrm{~Hz}$. Thresholds were measured with several interaural time delays (ITDs) of the noise ranging from 0 to $2470 \mu$ s in steps of $130 \mu$ s. The bands of noise had a duration of $300 \mathrm{~ms}$ including 20-ms, cosine-squared on/off ramps and had a spectrum level equivalent to $60 \mathrm{~dB}$ SPL. The duration of the signals was $250 \mathrm{~ms}$ (including 20$\mathrm{ms}, \operatorname{cosine}^{2}$, on/off ramps) and the signals were temporally centered in the band of noise. The signals were always presented diotically (So) resulting in a binaural configuration commonly referred to as $\mathrm{N} \tau$ So.

All stimuli were digitally generated utilizing a $32-\mathrm{kHz}$ sampling rate and were played out using 16-bit D/A converters. Before each block of trials that led to an estimate of threshold, a 4-s long circular buffer of Gaussian noise was calculated and independent, 300-ms long, samples of that noise were drawn randomly to produce the noise stimulus in each observation interval. The buffers of noise were constructed by first drawing independent samples from a Gaussian distribution and then applying a discrete Fourier transform to those samples which resulted in a spectrum having a spacing of $0.25 \mathrm{~Hz}$ between components. The spectral components outside the desired passband were set to zero and an inverse Fourier transform yielded the desired 4-s-long circular buffer of band-limited Gaussian noise. Noises constructed in this manner have a long-term spectrum with extremely steep spectral edges.

The testing of the conditions was completely randomized, except that the estimates of thresholds obtained with signal frequencies of 450 and $525 \mathrm{~Hz}$ in the presence of the higher-frequency noise were collected in pairs. Three of the authors (AK, SP, and MH) participated in the experiment. All listeners had normal hearing and had extensive experience in formal binaural listening tasks. Initial testing was conducted in single-walled, sound-attenuating chambers housed in laboratories at IPO, The Netherlands, using Beyer DT 880 headphones. Follow-up experiments were conducted at IPO and at the psychoacoustics laboratory at the University of Connecticut Health Center using TDH-39 earphones at both institutions.

\section{RESULTS}

The behavioral thresholds are plotted in Fig. 1 along with curves representing the cosine functions that best fit the respective sets of data. In Fig. 1, panels (a) and (b), respectively, contain data obtained when the frequency was $525 \mathrm{~Hz}$ (closed triangles) or $450 \mathrm{~Hz}$ (closed squares) and when the noise had components ranging from 600 to $1100 \mathrm{~Hz}$. Panel (c) of Fig. 1 contains data obtained when the frequency of the signal was $525 \mathrm{~Hz}$ and the components of the noise ranged from 0 to $450 \mathrm{~Hz}$ (closed circles).

The data points represent means calculated across the three listeners and the error bars represent the standard errors of those means. Because we are principally interested in the patterning of the data as a function of ITD, individual differences in overall threshold were removed during the calculation of the standard errors in each plot. This was done by subtracting the mean of the data of each listener from the grand mean, in each signal condition, respectively.

Let us first consider data obtained with the higherfrequency band of noise [Fig. 1, panels (a) and (b)]. In both cases, the thresholds vary with ITD in a manner that appears to be approximately cosinusoidal. Note that the patterns of thresholds obtained with the two signals are different, especially for ITDs greater than $1000 \mu \mathrm{s}$. When the frequency of the signal was $525 \mathrm{~Hz}$ [panel (a)], thresholds increased more steeply for delays greater than $1000 \mu$ s than did the thresholds obtained when the signal was $450 \mathrm{~Hz}$ [panel (b)]. In addition, when the signal frequency was $525 \mathrm{~Hz}$, a maximum in threshold occurred when the ITD was $1690 \mu$ s and performance improved as ITDs were increased to $2470 \mu \mathrm{s}$. In contrast, when the signal frequency was $450 \mathrm{~Hz}$, thresholds increased for ITDs of up to $2080 \mu$ s or so.

We examined periodicities within the data using the simplifying assumption that the thresholds varied as a cosinusoidal function of ITD. We found the best fitting cosine function (based on a least-squares criterion) for the data shown in panels (a) and (b). Amplitude and frequency were allowed to vary, but phase was constrained to be zero. This was done because a value of zero ITD would be expected to lead to the poorest performance (i.e., a maximum in threshold) given that the signals were presented in the So configuration. That is, the zero ITD in the noise results in an NoSo condition, a binaural configuration that does not contain interaural differences.

When the frequency of the signal was $525 \mathrm{~Hz}$ [panel (a)], the data were best fit by a cosine function having a frequency of $540 \mathrm{~Hz}$ and $84 \%$ of the variance in the data was 

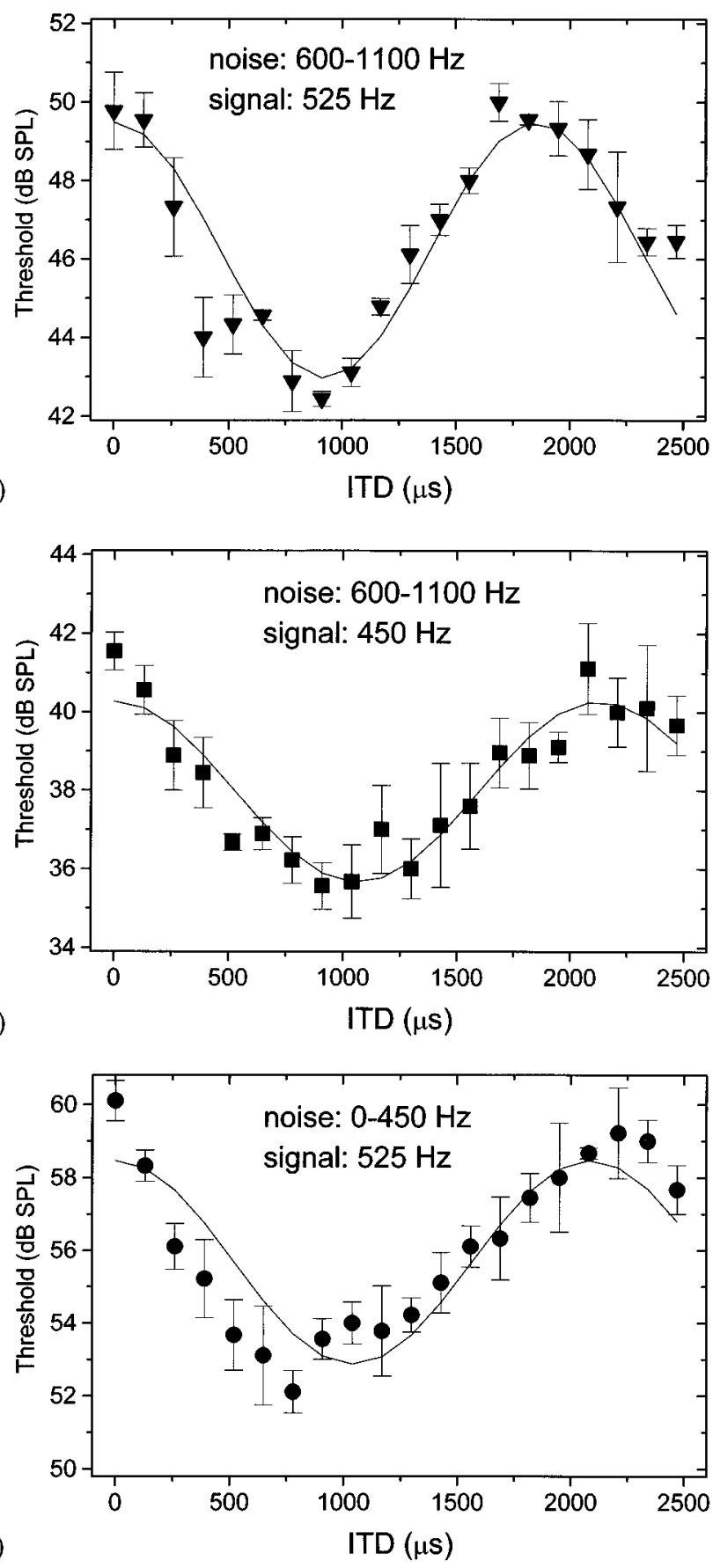

(c)

FIG. 1. Average thresholds of the three listeners as a function of ITD of the noise. Error bars indicate across-listener standard errors computed after differences in overall threshold were removed (see text). The data points in panels (a) and (b) were obtained when the spectral content of the noise ranged from 600 to $1100 \mathrm{~Hz}$ and when the frequency of the signal was either $525 \mathrm{~Hz}$ [panel (a), closed triangles] or $450 \mathrm{~Hz}$ [panel (b), closed squares]. The data points in panel (c) (closed circles) were obtained when the spectral content of the noise ranged from 0 to $450 \mathrm{~Hz}$ and the frequency of the signal was $525 \mathrm{~Hz}$. Solid lines in each panel indicate cosine functions that best fit the data.

accounted for by that function. That function is represented by the solid line in panel (a). Note that this best fitting frequency of $540 \mathrm{~Hz}$ is well below the spectral content of the noise and close to the frequency of the signal. This outcome is consistent with the hypothesis that the signal was being masked by aural distortion products, an interpretation that will be discussed in detail after other data are presented.

When the frequency of the signal was $450 \mathrm{~Hz}$ [Fig. 1, panel (b)], the data were best fit by a cosine function having a frequency of $469 \mathrm{~Hz}$ (solid line). That frequency accounted for $87 \%$ of the variance in the data. Although the spectral content of the noise was the same as when the frequency of the signal was $525 \mathrm{~Hz}$ [panel (a)], the frequency that best fits the data is now $71 \mathrm{~Hz}$ lower than before. As before, the best fitting frequency is well below the spectral content of the noise and close to the frequency of the signal. Overall, based on the frequencies that best fit the data, the patterning of the data in panels (a) and (b) of Fig. 1 is consistent with the hypothesis that the signals were masked by aural distortion products.

Let us now, using the same fitting methods as before, consider how well the data are fit by other frequencies besides the best fitting ones. This will provide an indication of how robust the data are in terms of being characterized by the single cosine functions shown in panels (a) and (b) of Fig. 1. Panel (a) of Fig. 2 shows the percentages of variance accounted for in the data presented in panels (a) and (b) of Fig. 1 as a function of the frequency used to fit the data. Note that the frequencies which account for the largest percentages in the data are clearly different for the two signal frequencies, despite the fact that the external or physical noise was the same in the two conditions. Also note that specification of a spectral region rather than a particular frequency appears to be a more accurate description of the quality of fits to the data by single cosine functions. Essentially identical amounts of variance were accounted for by a small band of frequencies surrounding the best fitting one. Still, it is clearly the case that the spectral regions that provide the best fits to the data are those that were expected to provide onfrequency masking due to aural distortion products.

Let us now turn to the data obtained with the lowerfrequency band of noise [Fig. 1, panel (c)]. The reader is reminded that the frequency of the signal $(525 \mathrm{~Hz})$ in this condition was above the spectral content of the noise, which ranged from 0 to $450 \mathrm{~Hz}$.

The behavioral thresholds in panel (c) vary as a function of ITD, indicating that interaurally delaying a noise can differentially affect the detectability of a signal placed spectrally above the highest frequencies in the noise. The maximum release from masking, about $8 \mathrm{~dB}$, occurred when the ITD was $780 \mu$ s. The solid line in Fig. 1, panel (c), represents the best fitting cosine function which, in this case, had a frequency of $479 \mathrm{~Hz}$ and accounted for $79 \%$ of the variance in the data. A frequency of $479 \mathrm{~Hz}$ is $29 \mathrm{~Hz}$ above the highest components of the noise and $46 \mathrm{~Hz}$ below the frequency of the signal.

Panel (b) of Fig. 2 shows the percentages of variance accounted for the data in panel (c) of Fig. 1 as a function of the frequency used to fit the data. Frequencies in the region between about 450 and $510 \mathrm{~Hz}$ account for between $72 \%$ and $79 \%$ of the variance of the data. In this case, the best fitting cosine functions are well below the signal frequency and slightly above the highest components of the noise. This outcome, taken in conjunction with the data discussed earlier, suggests that different processes or mechanisms may mediate 


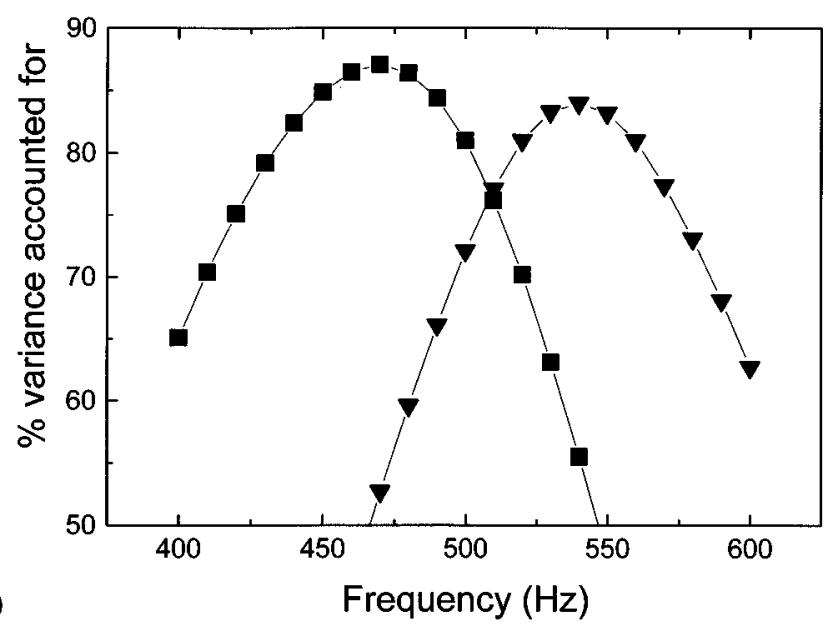

(a)

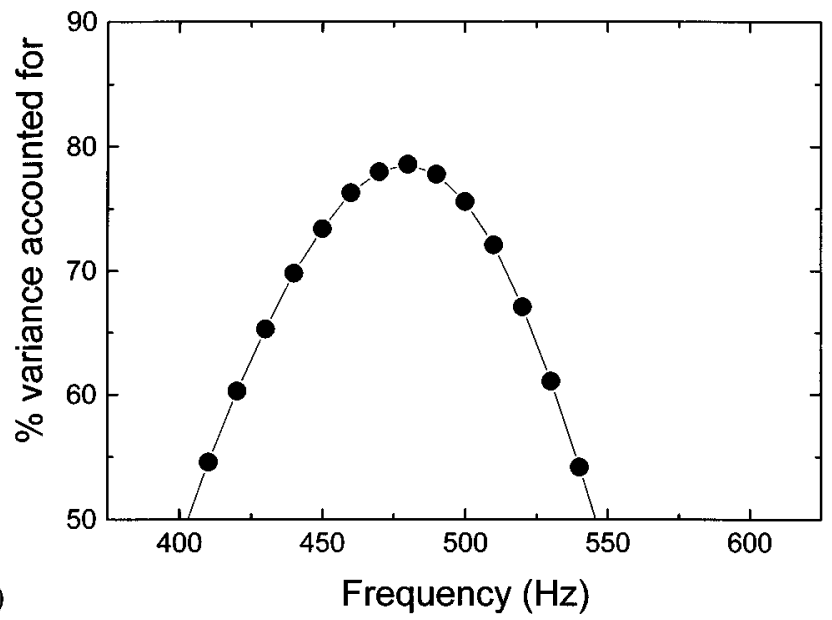

FIG. 2. The percentage of variance in the data of Fig. 1 accounted for by fitted cosines as a function of the frequency of the fitting cosine functions. In panel (a), this value is shown for the thresholds obtained when the spectral content of the noise ranged from 600 to $1100 \mathrm{~Hz}$. The two different curves represent the two different signal frequencies: $450 \mathrm{~Hz}$ (closed squares) and $525 \mathrm{~Hz}$ (closed triangles). In panel (b), the percentage of variance accounted for is shown for the thresholds obtained when the spectral content of the noise ranged from 0 to $450 \mathrm{~Hz}$ and the signal frequency was $525 \mathrm{~Hz}$ (closed circles).

binaural detection depending on whether the frequency of the signal is below or above the spectral content of the external noise.

Although the data presented in the three panels of Fig. 1 are well fit by cosine functions, close visual inspection indicates that there are systematic departures from the best fitting cosine functions, especially for the thresholds obtained with the smaller values of ITD. Among the most striking examples are the thresholds in Fig. 1, panel (c), for TDs ranging from 260 to $780 \mu$ s. Those data clearly suggest a much more rapid decline in threshold than that described by the best fitting function. We were also concerned that those data appear to have a second maximum at an ITD of about 2210 $\mu \mathrm{s}$ that is located beyond the maximum displayed by the best fitting cosine function of $479 \mathrm{~Hz}$. These aspects of the data are addressed next in Sec. III.

\section{DISCUSSION}

We begin by discussing the results in terms of Durlach's equalization-cancellation model (Durlach, 1963, 1972). In an
$\mathrm{N} \tau$ So condition such as those investigated here, equalization is accomplished via internal delays that compensate for the ITD in the noise. The stimulus condition is thereby transformed into a new configuration in which the noise is, ideally, interaurally identical (No). After equalization, the signal contains a time-delay equal to the internal delay required to equalize the noise. In this manner, the $\mathrm{N} \tau$ So condition becomes, after equalization, effectively equivalent to $\operatorname{NoS} \tau$, where $\tau$ is the time delay of the signal corresponding to the internal delay required to match the external delay of the noise.

Now, in our experiment, if the listeners were able to match (internally) the ITD of the noise, then one would expect that $\mathrm{N} \tau \mathrm{S}$ o thresholds would vary in the same manner as would thresholds obtained in an $\operatorname{NoS} \tau$ condition with the same signal frequency. In order to see if this were true, we retested the same listeners in the three main conditions of our original experiment utilizing an $\mathrm{NoS} \tau$ stimulus configuration. For each signal frequency, we used several ITDs that were equivalent to phase shifts of up to $180 \mathrm{deg}$. In addition, for reasons discussed later, we also obtained data using an $\mathrm{N} \pi \mathrm{So}$ stimulus condition.

The new data, obtained in the $\operatorname{NoS} \tau$ condition, are plotted in the three panels of Fig. 3 as open symbols. The data represented by closed symbols are replotted from Fig. 1.

When the signal was spectrally below the noise [panels (a) and (b) of Fig. 3], thresholds obtained with ITDs of the signal greatly overlap thresholds obtained with ITDs of the noise, provided that the ITD was less than $500 \mu$ s or so. For larger values of ITD, thresholds obtained with ITDs of the signal are consistently lower than thresholds obtained with ITDs of the noise. Panel (c) of Fig. 3 contains similar trends, save for the fact that the thresholds obtained with ITDs of the signal and thresholds obtained with ITDs of the noise now overlap for ITDs of up to about $800 \mu \mathrm{s}$.

We believe that the overlapping of the thresholds in the $\mathrm{N} \tau \mathrm{So}$ and $\operatorname{NoS} \tau$ conditions is evidence that the external delays were equalized or matched by appropriate internal delays. This statement holds for the noise components originating externally and for noise components generated internally by auditory nonlinearities. In contrast, the nonoverlapping of $\mathrm{N} \tau$ So and $\operatorname{NoS} \tau$ thresholds for larger delays (delays between 500 and $1000 \mu$ s or so) is taken as evidence that the external delays of the noise were not matched internally. This suggests that there is a limitation on the size of the ITD that can be matched internally. If that were true, then how does one explain the pattern of thresholds obtained with ITDs too large to be matched internally? Our hypothesis is that, for ITDs too large to be matched internally, detection is based on the interaural correlation coefficient. The interaural correlation coefficient is the value of the interaural correlation function at lag zero. Therefore, decisions based on it do not require equalization accomplished via the delay line. Our hypothesis differs from that made by Durlach (1972) and others, who assume that detection depends on the "best available internal delay" independent of whether that delay completely equalizes the external delay.

This line of argument leads us to look for two distinct regions in the data. In the region of "small" ITDs (those that 


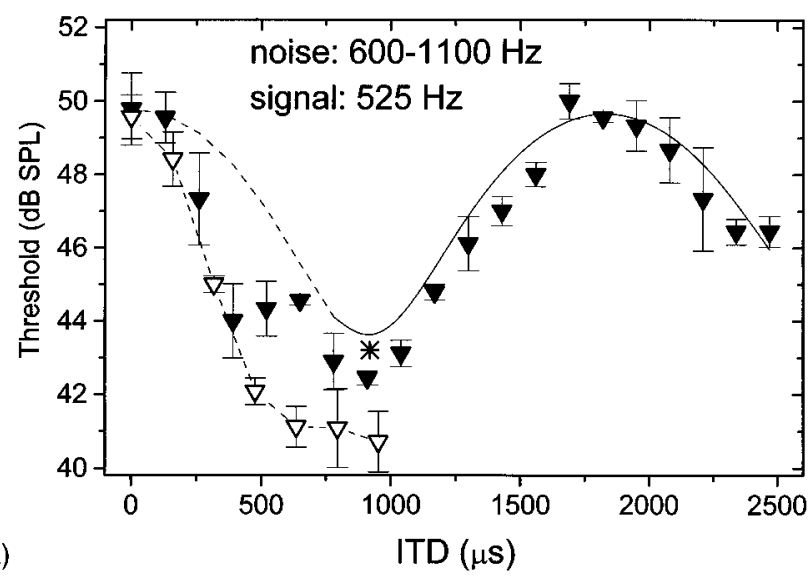

(a)

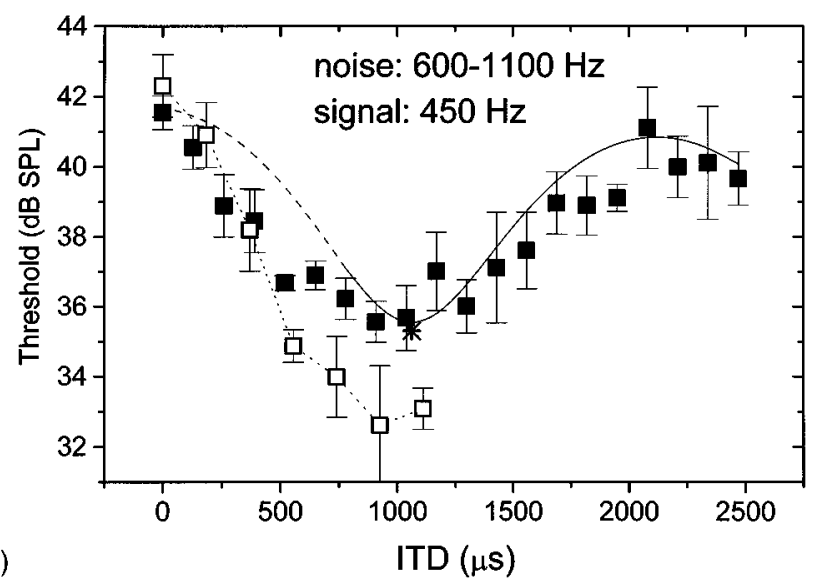

(b)

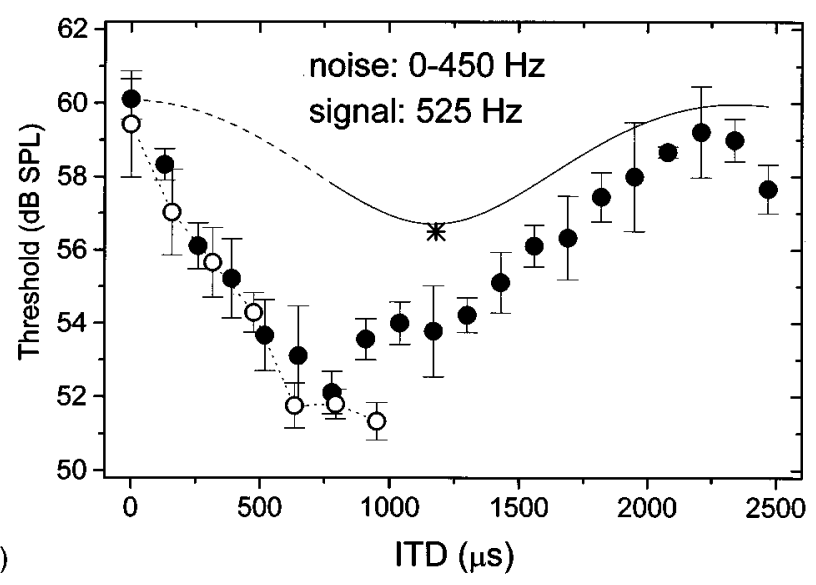

FIG. 3. Open symbols in panels (a)-(c) indicate $\operatorname{NoS} \tau$ thresholds as a function of the ITD of the signal. Closed symbols in panels (a)-(c) indicate thresholds obtained in the corresponding spectral conditions using the $\mathrm{N} \tau \mathrm{So}$ configuration (these data are replotted from Fig. 1). Different panels correspond to different spectral conditions: the data in panels (a) and (b) were obtained with the spectral content of the noise ranging from 600 to $1100 \mathrm{~Hz}$ (and with signal frequencies of 525 and $450 \mathrm{~Hz}$, respectively) and the data in panel (c) were obtained with the spectral content of the noise ranging from 0 to $450 \mathrm{~Hz}$ (and a signal frequency of $525 \mathrm{~Hz}$ ). Solid lines indicate predictions based on the interaural correlation of an interaurally delayed noise stimulus for ITDs greater than $700 \mu$ s. The dashed lines represent extensions of the predictions to include ITDs less than $700 \mu \mathrm{s}$. N $\pi$ So thresholds are indicated by asterisks.

can be matched internally), the pattern of thresholds would reflect the frequency of the signal. In the region of "large" ITDs (those that cannot be matched internally), the pattern of thresholds would reflect the periodicity in the autocorrelation of the noise that is responsible for the masking.

We now evaluate whether $\mathrm{N} \tau$ So thresholds obtained with ITDs presumably too large to be matched internally can be explained using the interaural correlation coefficient. We will begin with the data obtained when the frequency of the signal was below the spectral content of the noise. We assume that masking in this case was due to "on-frequency" aural distortion products and that the patterning of the data will reflect this. The methods used to evaluate this assumption were a bit complicated. We inspected the autocorrelation function of a $100-\mathrm{Hz}$-wide band of noise centered on the frequency the signal. The bandwidth of $100 \mathrm{~Hz}$ was chosen to approximate the bandwidth of noise at the output of a critical band at these low signal frequencies. The spectrum of the bands of noise contained a tilt (a spectral slope of 0.11 $\mathrm{dB} / \mathrm{Hz}$ ) favoring the higher frequencies. That value of spectral slope was determined by taking the 8 - $\mathrm{dB}$ difference in detection threshold (obtained with an ITD of $0 \mu \mathrm{s}$ ) for the two signal frequencies placed spectrally below the noise and dividing that difference in threshold by the $75-\mathrm{Hz}$ difference in the frequency of the signals. We made the reasonable assumption that the 8-dB difference in diotic (NoSo) thresholds for the two signal frequencies occurred because the noise power responsible for the masking differed by $8 \mathrm{~dB}$ across the two signal frequencies. This type of spectrum, "tilted" toward the higher frequencies, is consistent with the literature concerning aural distortion products (e.g., Goldstein, 1967; van der Heijden and Kohlrausch, 1995). Because of the spectral tilt, the autocorrelation function of the 100$\mathrm{Hz}$-wide band of noise, although centered on the frequency of the signal, is quasi-periodic with a frequency slightly higher than the frequency of the signal.

We evaluated the autocorrelation functions of the two noises for ITDs greater than $700 \mu$ s, a value chosen by considering all the data. The process involved recasting $\mathrm{N} \tau \mathrm{So}$ conditions into $\mathrm{N} \rho \mathrm{So}$ conditions, with $\rho$ being the interaural correlation of noise stemming from aural distortion products. In order to do so, we had to obtain additional data using an $\mathrm{N} \pi$ So stimulus configuration. These $\mathrm{N} \pi \mathrm{So}(\rho=-1)$ thresholds, in combination with the NoSo $(\rho=+1)$ thresholds obtained in the main experiment, served as endpoints in the function relating $\mathrm{N} \rho \mathrm{So}$ detection thresholds to $\rho$. In order to obtain predictions of thresholds for intermediate values of $\rho$, we utilized the interpolation method recently described by van der Heijden and Trahiotis (1996).

The solid lines in panels (a) and (b) of Fig. 3 represent predictions obtained for ITDs $\geqslant 700 \mu$ s using the interaural correlation coefficient of the tilted-spectrum bands of noise representing aural distortion products as discussed earlier. The reader is reminded that these predictions were made assuming that external ITDs were not matched internally. The correspondence between the predictions and the data appears to be quite good in both cases. When the signal frequency was $525 \mathrm{~Hz}$ [panel (a)], the amount of variance in the data accounted for by the predictions was $87 \%$. When the signal frequency was $450 \mathrm{~Hz}$ [panel (b)], the amount of variance in the data accounted for by the predictions was $75 \%$.

A corollary of our theoretical position is that extending 
the predictions to ITDs less than $700 \mu$ s (the ITDs we have argued are internally matched) would reduce the amount of variance accounted for in the data. Indeed, as shown by the dashed line extensions to our predictions in Fig. 3, the predictions for the smaller ITDs would be quite poor. When we included those values of ITD in the analysis, the amount of variance accounted for decreased and was $67 \%$ and $65 \%$, for the $525-\mathrm{Hz}$ and the $450-\mathrm{Hz}$ signals, respectively.

Overall, our analyses strongly suggest that aural distortion products are responsible for the masking that occurred when the frequency of the signal was below the spectral content of the noise. In our judgment, the predictions based on aural distortion products are remarkably good, especially when it is considered that the predictions were made by taking into account: only two behavioral thresholds and logical arguments concerning the properties of the aural distortion products assumed to be responsible for the masking.

As a check on the sensitivity of our analysis, we also investigated the autocorrelation function assuming a critical bandwidth of $200 \mathrm{~Hz}$ rather that $100 \mathrm{~Hz}$. The amount of variance accounted for when the frequency of the signal was $525 \mathrm{~Hz}$ dropped to $72 \%$. The amount of variance accounted for when the frequency of the signal was $450 \mathrm{~Hz}$ dropped to $61 \%$. We take this as evidence that the first analysis, using the 100-Hz-wide critical bandwidth, was not a fortuitous success and that our data and methods have sufficient precision.

We now consider predictions for the case when the $525-\mathrm{Hz}$ signal was spectrally above the components of the physical noise which ranged from 0 to $450 \mathrm{~Hz}$. We investigated the interaural crosscorrelation of a $50-\mathrm{Hz}$-wide, rectangular, band of noise centered at $425 \mathrm{~Hz}$. This band of noise was chosen because we assumed that masking was produced by frequencies at the upper edge of the noise and that such a band of noise would not be removed by an internal filter centered on $525 \mathrm{~Hz}$, the signal frequency.

Predictions obtained using the general method described above are shown as the solid line in panel (c) of Fig. 3. Note that, in this case, the predicted thresholds are a poor fit to the data and are consistently higher than the obtained thresholds. In fact, using the mean as a predictor would provide a better fit to the data.

It is not the case that our assumption concerning which frequencies produced masking is responsible for the poor predictions obtained in this condition. On the contrary, the data obtained with ITDs greater than $700 \mu$ s are extremely well fit by single cosine functions having a frequency within the upper region of the band of noise. In fact, cosine functions having a frequency in the range of $420-450 \mathrm{~Hz}$, the uppermost region of the noise, each accounted for more than $90 \%$ of the variance in the behavioral data. At the same time, a cosine function of $525 \mathrm{~Hz}$, the frequency of the signal, accounted for less than $60 \%$ of the variance in the data collected with ITDs greater than $700 \mu$ s. Therefore, the inability to predict thresholds when the frequency of the signal was above the spectral content of the noise is not due to the periodicities in the data being somehow incompatible with the spectral region assumed to be responsible for the masking.

Our interpretation of the data is predicated on cancella- tion of noise for the smaller delays that can be internally matched and on the use of the correlation coefficient (i.e., the correlation function at lag zero) for larger delays that cannot be internally matched. Of course, it is possible that binaural detection for "in-between" values of external delay may depend upon a combination of both modes of processing and/or a differential weighting of internal delays depending on their usefulness. In addition, our interpretations do not explicitly consider the potential use of "slipped cycles" for detection when external delays are large enough so that complete cancellation is impossible. As discussed by Rabiner et al. (1966), especially on pages 69 and 70, explanations of binaural detection data depending upon the use of slipped-cycle information involve a myriad of complex issues and data. At this time, we cannot provide an analysis of our data that incorporates, let alone resolves, the complex issues discussed by Rabiner et al. However, we believe that future data obtained utilizing very large delays (representing multiples of the periods of the signals) in our paradigm with masking produced by aural distortion products may permit the use of information in slipped cycles to be evaluated. Still, it appears to us that our analyses are an appropriate beginning toward the understanding of binaural detection data obtained under conditions where aural distortion products could be responsible for the masking.

We now present arguments that indicate that there is some other, unknown, factor that plays a role in binaural detection when the signal frequency is above the masking noise. Recall that, in order to make predictions for several values of interaural correlation via our interpolation method, we had to measure thresholds using an $\mathrm{N} \pi$ So configuration. This was done for all three of our main experimental conditions. Those thresholds, which are represented by asterisks in panels (a)-(c) of Fig. 3, were very close to their N $\tau$ So counterparts when the frequency of the signals was lower than the spectral content of the noise [Fig. 3, panels (a) and (b)].

Note, however, that the $\mathrm{N} \pi$ So threshold measured with the frequency of the signal above the spectral content of the masker [Fig. 3, panel (c)], is $3 \mathrm{~dB}$ higher than its $\mathrm{N} \tau \mathrm{So}$ counterpart. As a consequence, when the frequency of the signal was above the spectral content of the noise, the MLD between $\mathrm{N} \pi$ So and NoSo conditions is slightly less than 4 $\mathrm{dB}$. In contrast, the MLD between the equivalent $\mathrm{N} \tau \mathrm{So}$ conditions (i.e., an ITD of about $1000 \mu \mathrm{s}$ ) and the NoSo conditions is about $7 \mathrm{~dB}$. This is a very important difference. $A$ priori, one would expect that $\mathrm{N} \pi$ So thresholds, for which the interaural correlation of the noise is -1 , would be equal to, or lower than, thresholds obtained with any other values of interaural correlation of the masker. This means that the variations in the thresholds obtained when the frequency of the signal was above the spectrum of the noise cannot be explained solely in terms of the interaural correlation of the masker. This interpretation comes from relations among the data and does not entail assumptions concerning which spectral components contributed to the masking.

At this time, we have no explanation for the masking that occurred when the signal was spectrally above the noise and when the ITD of the noise was presumably too large to be equalized. In future investigations, we plan to determine 
whether, and to what degree, binaural interference effects and binaural masking effects may combine or interact to determine detectability for signals placed spectrally above maskers and/or interferers. Binaural interference refers to degradations in the ability to detect or discriminate interaural differences in cases where masking effects can be ruled out. As discussed by Bernstein and Trahiotis (1995), binaural interference effects are often asymmetric in that low-frequency interferers affect high-frequency targets more than highfrequency interferers affect low-frequency targets. At this time, it appears not too far-fetched to speculate that noises containing large ITDs cannot be equalized and, therefore, cannot be canceled. Consequently, they may remain to affect detectability via binaural interference.

In summary, we have presented data suggesting that aural distortion products that are evoked by a band of noise have the ability to produce binaural masking of tonal signals having a frequency below the spectral content of the noise. The detectability of such signals is well accounted for by considering the expected interaural correlation of aural distortion products evoked by interaurally delayed noise. We conclude that aural distortion products can produce binaural as well monaural masking effects (e.g., van der Heijden and Kohlrausch, 1995). On the other hand, data obtained with tonal signals having a frequency above the spectral content of the noise are not satisfactorily accounted for by considering the interaural correlation of the noise. It appears that another factor, perhaps binaural interference, limits binaural detectability for signals placed spectrally above masking noise.

\section{ACKNOWLEDGMENTS}

The authors thank Dr. Les Bernstein for his careful reading of several drafts of this manuscript. His many sugges- tions are appreciated. We also thank Dr. H. Steven Colburn, an identified reviewer, who correctly, yet supportively, stated that our original discussion section needed severe reorganization. Supported by research Grant No. NIH DC-00234 from the National Institute on Deafness and Other Communication Disorders, National Institute of Health.

Bernstein, L. R., and Trahiotis, C. (1995). "'Binaural spectral interference in detection and discrimination paradigms," in Advances in Hearing Research: Proceedings of the 10th International Symposium on Hearing, edited by G. A. Manley, G. M. Klump, C. Koppl, H. Fastl, and H. Oekinghaus (World Scientific, Singapore).

Bilger, R. C., and Hirsch, I. J. (1956). "Masking of tones by bands of noise," J. Acoust. Soc. Am. 28, 623-630.

Durlach, N. I. (1963). "Equalization and cancellation theory of binaural masking-level differences," J. Acoust. Soc. Am. 35, 1206-1218.

Durlach, N. I. (1972). "Binaural signal detection: Equalization and cancellation theory," in Foundations of Modern Auditory Theory, Volume II, edited by J. V. Tobias (Academic, New York), pp. 365-466.

Goldstein, J. L. (1967). “Aural combination tones,'” J. Acoust. Soc. Am. 41, 676-689.

Hall, J. W., Tyler, R. S., and Fernandez, M. A. (1983). "Monaural and binaural auditory frequency selectivity resolution measured using bandlimited noise and notched-noise masking," J. Acoust. Soc. Am. 73, 894898.

Langford, T. L., and Jeffress, L. A. (1964). " Effect of noise crosscorrelation on binaural signal detection,' J. Acoust. Soc. Am. 36, 1455-1458.

Levitt, H. (1971). "Transformed up-down methods in psychoacoustics," J. Acoust. Soc. Am. 49, 467-477.

McFadden, D., Russell, W. E., and Pulliam, K. A. (1972). "Monaural and binaural masking patterns for a low-frequency tone,' J. Acoust. Soc. Am. 51, 534-543.

Rabiner, L. R., Laurence, C. L., and Durlach, N. I. (1966). "Further results on binaural unmasking and the EC model," J. Acoust. Soc. Am. 40, 6270 .

van der Heijden, M., and Kohlrausch, A. (1995). "The role of distortion products in masking by single bands of noise,' J. Acoust. Soc. Am. 98, $3125-3134$.

van der Heijden, M., and Trahiotis, C. (1996). "A new way to account for binaural detection as a function of interaural noise correlation," J. Acoust. Soc. Am. (accepted for publication). 